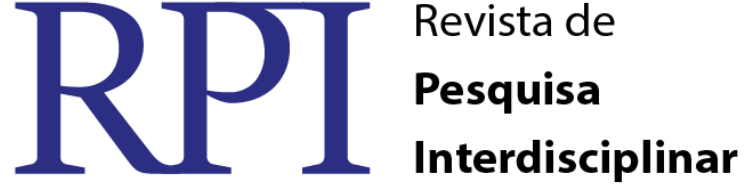

\section{O YOUTUBE COMO FERRAMENTA PEDAGÓGICA EM SALA DE AULA: UMA PRÁTICA DE LETRAMENTO}

\author{
Adriana Moreira de Souza Correa (UFCG) \\ Hérica Paiva Pereira (UFCG)
}

\begin{abstract}
RESUMO
Através desta pesquisa constatamos a relevância de trabalhar atividades que visem o multiletramento no âmbito do ensino de uma segunda língua, que para efeito desta pesquisa, trata-se da Língua de Sinais. Observamos que o desenvolvimento das práticas de letramentos dá margem para que o aluno perceba que, além da superfície do texto, existem informações que podem ser inferidas apenas no contexto da informação, como por exemplo: o conhecimento da cultura e como a informação foi passada. Como objetivo apresentamos o YouTube como ferramenta que favorece o multiletramento a medida que colabora para a compreensão da Língua de Sinais, no que diz respeito à estrutura, ao léxico e à cultura que é expressa por essa língua. Para isso buscamos identificar a relação existente entre os textos produzidos na Libras e disponibilizados no YouTube com os conhecimentos externos à língua que contribui para a compreensão do que foi enunciado. Este meio de comunicação permite que os gêneros textuais sejam compreendidos na sua estrutura e na sua função comunicativa, não se limitando à compreensão de estruturas linguísticas isoladas, já que está pautada no contexto da enunciação. Isso quer dizer que, ao fundamenta-se em textos reais, o aluno terá um modelo para reformular os seus próprios textos em uma segunda língua e, portanto, significativa para ele. Diante do exposto a nossa pesquisa está embasada nos estudos de Rojo (2012), Kleiman (2005), Almeida et. al.(2015), Skliar (2001) e Schallenberger (2012). Enquanto, no que se refere à metodologia, constituise em uma análise bibliográfica que busca identificar os dados qualitativos referentes à temática abordada.
\end{abstract}

Palavras - Chave: Multiletramentos. Ensino de Libras. YouTube.

\section{YOUTUBE AS EDUCATIONAL TOOL IN THE CLASSROOM: A LITERACY PRACTICE}

\begin{abstract}
Through this research we see the importance of working activities aimed at multiliteracy in teaching of a second language, which for purposes of this research, it is the Sign Language. We note that the development of literacies practices permit that the student realizes that besides the text surface, there is information that can be inferred only in the context of information, such as: knowledge of the culture and how the information was passed. It is destined to present the YouTube as a tool that favors the multiliteracies the measure which contributes to the understanding of sign language, with respect to the structure, vocabulary and culture that is expressed by this language. For this we seek to identify the relationship between the texts produced in Libras and made available on YouTube with external knowledge to the language that contributes to the understanding of what was stated. This medium allows that the textual genres be understood in its structure and in its communicative function, not limited to the understanding of isolated linguistic structures, since it is guided in the context of enunciation. This means that, based on actual texts, the student will have a model to reformulate their own texts in a second language and therefore meaningful to him. Given the above our research is grounded in Rojo's (2012), Kleiman's (2005), Almeida et. al.'s (2015), Skliar's (2001) and Schallenberger's (2012) studies. Meanwhile, as regards the method, it is in a literature review seeks to identify the qualitative data regarding the selected theme.
\end{abstract}


KeywordS: Multiliteracies. Libras teaching. YouTube.

\section{INTRODUÇÃO}

Diante da sociedade que valoriza as novas tecnologias, a educação não pode ficar indiferente ao uso destes recursos, especialmente ao se tratar de textos produzidos em línguas visuais espaciais que requerem um suporte que permita a visualização de textos em vídeos. Diante disso, necessitamos buscar ferramentas que possibilitem a compreensão dos alunos diante dos conteúdos que se deseja expressar, sendo estes mediados pelas tecnologia de uso corrente na sociedade atual. Neste trabalho, apresentamos o YouTube como uma ferramenta adequada para a compreensão, construção e disponibilização de textos em Língua de Sinais.

O principal objetivo é apresentar o YouTube como ferramenta utilizada nos Multiletramentos que colaboram para a compreensão da Língua de Sinais, no que diz respeito à estrutura, ao léxico e à cultura que é expressa por essa língua.

Para fundamentar a pesquisa, nos embasamos, principalmente, nos estudos de Rojo (2012), Kleiman (2005), Almeida Et. Al.(2015), Skliar (2001) E Schallenberger (2012). Enquanto a metodologia é uma análise bibliográfica que gerou um estudo qualitativo da temática apresentada.

A construção do texto está composta por três capítulos, sendo que o primeiro é a introdução; o segundo aborda os multiletramentos e o YouTube, e na sequencia apresentamos uma discussão acerca da importância do trabalhar com os vídeos, disponibilizados pelo YouTube, como uma ferramenta que contribui para a construção do conhecimento na segunda língua.

\section{Multiletramento em sala de aula}

\section{Um novo modo de pensar a sala de aula através dos multiletramentos}

Um dos grandes desafios que, nós professores, encontramos hoje é inserir a criança, o jovem e o adulto na cultura letrada, ou seja, trazer para a escola e para a universidade o aluno com toda a sua bagagem cultural, seus valores e conhecimentos. Nesta perspectiva, Kleiman RPI Revista de Pesquisa Interdisciplinar, Cajazeiras, v. 1, Ed. Especial, 381 - 389, set/dez. de 2016. 
(2007), defende que o ambiente escolar é o lugar propício para esta troca em que ocorrem práticas discursivas através de diferentes atividades inseridas em contextos vários. Não obstante isso, ela reconhece que, um dos grandes obstáculos para que isso ocorra, são as aulas tradicionais, ainda fortemente arraigadas na prática de muitos professores que, consideram "a aprendizagem da leitura e produção textual como aprendizagem de competências e habilidades individuais" (p.4).

Para a autora estas práticas são fundamentais no exercício pedagógico de cada docente que quer desenvolver atividades com os gêneros textuais utilizados no uso cotidiano do aluno e, portanto significativo para ele. Ainda para a autora a prática social deve nortear o processo metodológico na construção da intervenção pedagógica que o professor realiza para desenvolver os diferentes conhecimentos do aluno.

Nesta perspectiva, entendemos a urgência em rever nossa prática pedagógica em sala de aula, ao realizar nossos planejamentos diários de forma flexível a fim de encontrar sempre novos caminhos que conduzam a uma melhor aprendizagem de nossos alunos.

De acordo com os estudos de Rojo (2012), um grupo de pesquisadores dos Estados Unidos da América, no ano de 1996, apresentou um manifesto intitulado "A pedagogia dos multiletramentos - desenhando futuros sociais". A intenção do grupo era de adicionar nos currículos uma nova pedagogia com o uso de novos letramentos que envolvesse, não somente as novas tecnologias de informação e comunicação, mas também as diferentes “culturas já presentes nas salas de aula de um mundo globalizado e caracterizada pela intolerância na convivência com a diversidade cultural, com a alteridade" (p.12). Nesta perspectiva, o termo multiletramento chegou com a intenção de trabalhar com os modernos fenômenos da linguagem através de um estudo multissemiótico ou mutimodal.

Ao referir-se a multiletramento, a autora afirma que este tem a característica de "um trabalho que parte das culturas de referência do alunado (popular, local, de massa) e de gêneros, mídias e linguagens por eles conhecidos, para buscar um enfoque crítico, pluralista, ético e democrático" (p.8). Para que isso ocorra faz-se necessário que cada docente ofereça as condições necessárias aos seus alunos, através de um processo de atividades que os envolva em práticas metacognitivas que lhes fazem pensar como atuar e encontrar respostas aos desafios apresentados.

No que diz respeito à multiplicidade de linguagens, conhecido também por multimodalidade ou multissemiose, característicos dos multiletramentos, nos detemos aqui à RPI Revista de Pesquisa Interdisciplinar, Cajazeiras, v. 1, Ed. Especial, 381 - 389, set/dez. de 2016. 
mídia como letramento midiático, que nos permite compartilhar com outras pessoas argumentos que nos interessam, através do uso de suportes como o computador, tablets e celulares. Estas são ferramentas utilizadas por quase toda a população e principalmente por crianças e jovens que se sentem atraídas pelo uso de textos que envolvem variadas linguagens, verbais e não verbais que requer certo conhecimento e prática para a sua compreensão, ou seja, de multiletramentos, para dar-lhes uma significação. Um exemplo deste tipo de atividade são os vídeos gravados por surdos e disponibilizados no YouTube em Língua de Sinais. Estes vídeos podem ser utilizados nas aulas de libras com o objetivo de transmitir aos alunos ouvintes momento de aprendizado da língua de forma viva e participativa, como é o caso da proposta de atividade que apresentaremos neste trabalho.

\section{O YouTube como ferramenta didática}

O site Youtube foi lançado em 2005, nos Estados Unidos da América, tendo como fundadores os estudantes de ciências da computação Steve Chen e Jawed Karin. Já o design, foi desenvolvido por Chad Hurley, que se ocupou em favorecer o compartilhamento de vídeos na rede. Atualmente, o site é utilizado em 75 países e é assistido por mais de um bilhão de usuários de 61 idiomas (YouTube, 2011).

Este site é um suporte que pode ser utilizado em diferentes dispositivos de comunicação, tais como celulares, tabletes, computadores, etc, desde que estejam conectados a uma rede e se tenha o conhecimento sobre o funcionamento deste suporte. Por meio deste portal de vídeos online podemos produzir e absorver conteúdos que podem ser compartilhados.

Atualmente, pela facilidade de acesso, este recurso vem sendo utilizado em vários espaços sociais, seja para divulgar informações científicas, como também os mais variados tipos de linguagens que circulam na sociedade, como por exemplo: receitas, piadas, entrevistas, etc. Neste mesmo suporte podemos encontrar as vídeo-aulas que podem ser acessadas em qualquer horário ou local. Elas possibilitam a incorporação de elementos relacionados à imagem, tais como figuras, gráficos animados e outros vídeos que tendem a ser mais atrativos que a exposição de conteúdos, servindo também para o ensino de Libras através de textos reais. Isso, sem deixar de ressaltar que os vídeos armazenados no YouTube podem ser compartilhados por outros aplicativos de grande uso na sociedade atual. Vejamos o que dizem os autores sobre o uso do YouTube como ferramenta no processo educacional:

RPI Revista de Pesquisa Interdisciplinar, Cajazeiras, v. 1, Ed. Especial, 381 - 389, set/dez. de 2016. 
Este site tornou-se fascinante, pois, expor a opinião, produzir informação, debates, conteúdos científicos, educacionais, humorístico entre outros fazem parte do que podemos chamar atualmente de cultura popular o que o torna útil para a compreensão das relações sociais, evolução das tecnologias e das mídias, auxiliando na práxis escolar (ALMEIDA et. al., 2015).

Nesta perspectiva, entendemos que o YouTube, além de possibilitar a comunicação entre as pessoas, é uma ferramenta que pode contribuir muito no processo de ensino aprendizagem. Isso ocorre, em função da possibilidade da percepção de traços, inferidos pelo contexto da fala e que são relevantes para a compreensão da língua.

\section{Discussões Sobre O Ensino De Libras Para Ouvintes, Mediada Por Vídeos Disponíveis No Youtube.}

Como vimos, as tecnologias de informação e comunicação estão presentes no cotidiano dos alunos na atualidade e, por esta razão, se convertem em instrumentos produtivos de pesquisa e disseminação do conhecimento. Tratando-se da disciplina de Língua Brasileira de Sinais - Libras, ofertada na modalidade presencial e que conta com uma professora ouvinte, deparamo-nos com o desafio de apresentar ao aluno também ouvinte, uma língua construída a partir de uma cultura visual. Neste sentido, faz-se necessário buscar estratégias para a apresentação dos aspectos culturais dos surdos e que permeiam os textos construídos nesta língua.

Considerando-se tratar de uma língua visual-espacial, os textos e os gêneros, em Libras, são construídos na perspectiva da imagem e do movimento e, por esta razão, os meios de veiculação da mensagem nesta língua adequam-se ao uso da comunicação. Schallenberger (2012) define a evolução dos meios de comunicação e informação que permitem o compartilhamento de vídeos como um dos grandes responsáveis pela valorização das produções dos surdos em Língua de Sinais. De acordo com o autor:

[...] o que o advento das tecnologias de registro e comunicação proporcionou foi a visualização de produções dos próprios surdos, em língua de sinais. [...] Este site, youtube, configura uma rede de troca de vídeos que possibilitou a abertura de novos caminhos na divulgação da língua de sinais, sendo uma

RPI Revista de Pesquisa Interdisciplinar, Cajazeiras, v. 1, Ed. Especial, 381 - 389, set/dez. de 2016. 
maneira de registrar produções como piadas, histórias, saberes da comunidade etc (SCHALLENBERGER, 2012, p. 78).

Nesta perspectiva, o site Youtube.com, que hospeda vídeos de livre acesso aos internautas, constitui-se em um meio propício para a seleção de textos reais, de diferentes gêneros que podem ser utilizados, analisados e ressignificados na aula de Libras.

Estes textos sinalizados, são tecidos por usuários surdos que têm na Libras a sua primeira língua e, por esta razão, trazem em si os modos de ser e de pensar deste grupo (PINHEIRO, 2011). Estes modos de ser e de pensar, para Skliar (2001), é definido através da experiência visual, que engloba as diferentes significações e valores coletivos culturais que por sua vez, são constituídos e refletem na Língua de Sinais. Isto nos remete às práticas de letramentos em que os conhecimentos prévios assim como a cultura de cada sujeito e seus valores são indispensáveis para a ampliação de novos significados.

Carlos Skliar destaca ainda que analisar o caráter visual das produções em Língua de Sinais possibilita a construção de "um significado crucial na comunicação e nos processos didáticos, curriculares e intelectuais mais amplos e não somente nas questões linguísticas" (SKLIAR, 2001, p. 103). Apesar do pesquisador tratar destas questões aplicadas à educação bilíngue para surdos, observamos também a necessidade da discussão deste aspecto no ensino da Língua de Sinais para os ouvintes. Desta forma, são trabalhadas simultaneamente, várias habilidades necessárias para a compreensão da mensagem que se deseja comunicar.

Primeiramente, identificamos que estes vídeos agem como instrumentos de aprendizado de informações que não se referem apenas ao vocabulário ou à organização gramatical de uma nova língua, tendo em vista que possibilitam a identificação e internalização dos traços paralinguísticos e dos extralinguísticos característicos das línguas naturais. Entendemos como traços paralinguísticos da língua de sinais as expressões faciais que podem indicar: o tom do discurso (inflamado, sereno e outros). Além disso temos o ritmo da sinalização e outras características que são inerentes às produções sinalizadas, ou seja, características que transcendem a sinalização e nos fazem perceber o significado não só pelo sinal, mas pela forma na qual estes são expressos. Já os traços extralinguísticos se referem àqueles que se tratam dos conhecimentos de mundo dos envolvidos no processo comunicativo (GUIMARÃES, 2009)..

Esses traços são relevantes nos estudos do letramento porque dizem respeito a tudo aquilo que vai mais além do que foi expresso em palavras ou em sinais, acrescentando ou RPI Revista de Pesquisa Interdisciplinar, Cajazeiras, v. 1, Ed. Especial, 381 - 389, set/dez. de 2016. 
modificando o sentido do que foi dito, dependendo dos conhecimentos culturais partilhados pelos sujeitos envolvidos na enunciação.

É importante salientar que os vídeos discutidos neste trabalho não se tratam das vídeoaulas para ensino da Libras, ou seja, dos vídeos produzidos para os cursos de Libras que apresentam-se na forma de vocabulário (onde o sinal é apresentado simultaneamente ou consecutivamente à sua tradução em Língua Portuguesa), mas aos vídeos que se caracterizam pela apresentação de um gênero como, por exemplo, o relato pessoal, a receita, contos etc.

Através destes vídeos, o aluno pode ainda entrar em contato com as diferentes formas de expressão e produção dos sinais, ou seja, dos alofones na Libras, que se tratam de formas de articulação diferenciadas de um mesmo fonema, mas que não geram modificação no significado do vocábulo (SANTOS; COSTA, 2012). Outra vantagem do uso de vídeos com sinalizações de surdos é a possibilidade de romper com a ideia de uma língua unificada à medida que se apresentam, contextualizadamente, "as variações regionais", presentes nas línguas, e por esta razão, são encontradas na Língua de Sinais. Karnopp; Klein;LunardLazzarin (2011, p. 21) asseguram que:

Quando referimos a Libras, isso não significa que ela seja utilizada da mesma forma por todos os surdos brasileiros. Como qualquer outra língua, ela está sujeita às variações regionais, adequando-se aos aspectos históricos, sociais e culturais das diferentes comunidades em que é utilizada. Da mesma forma, as produções artístico culturais sofrem influências destes aspectos.

Além de trabalhar a estrutura e o vocabulário presentes no texto, podemos ainda, através destes vídeos, discutir conceitos e visões acerca de como a comunidade surda se vê e como o ouvinte vê o surdo (PINHEIRO, 2011, p.29). Desta forma, a compreensão das diversas relações que o surdo mantém, com a surdez e com o mundo ouvinte, podem ser apreciadas, discutidas e compreendidas a partir de diferentes gêneros. Karnopp; Klein;Lunard-Lazzarin (2011, p. 24) afirmam que:

Os materiais analisados a partir dos vídeos coletados no YouTube demarcaram a circulação livre na internet de produções culturais surdas. Por meio dos registros visuais em categorias artísticas, como encenações, propagandas, piadas e demais manifestações de pessoas surdas.

RPI Revista de Pesquisa Interdisciplinar, Cajazeiras, v. 1, Ed. Especial, 381 - 389, set/dez. de 2016. 
Desta forma, observamos que este meio de comunicação aloja diferentes gêneros que servem para a compreensão do vocabulário contextualizado, a compreensão do conteúdo, a apreensão das características do texto em função da sua função significativa e serve como um modelo textual para a criação de outros textos, seja do mesmo gênero ou pela transformação de um gênero em outro, como ocorre no processo de retextualização apresentado por Dell'Isola (2007), apesar da autora tratar, nesta obra, de línguas orais-auditivas, observamos que a proposta pode ser produtiva na Língua de Sinais.

\section{Consideraçoes Finais}

Pudemos constatar que o trabalho com os Multiletramentos é uma resposta aos anseios, de muitos alunos, que esperam a inserção das novas tecnologias e mídias, utilizadas no cotidiano, na prática escolar. Esse permite um desenvolvimento integral do aluno, ao permitir que a escola traga, para o seu âmbito, todos os conhecimentos prévios do discente, como também seus valores e cultura.

Nesta perspectiva, o YouTube, apresenta-se como uma ferramenta ideal para que o aluno desenvolva uma visão mais crítica da relação entre língua e cultura, ou seja, dos traços que devem apresenta-se, de forma explícita, na língua e daqueles que são inferidos apenas na situação comunicativa. Este site disponibiliza textos em vídeos, favorecendo a identificação e a produção de materiais realizados por alunos ou professores, com a finalidade de selecionar conteúdos que favoreça a compreensão em Libras e a produção de novos textos que poderão ser disponibilizados na rede.

Pudemos constatar ainda que, trabalhar as práticas do letramento, significa ir mais além daquilo que o texto apresenta em sua superficialidade, porque ela nos permite investigar os elementos motivadores encontrados na produção do vídeo, ou seja, a sua função social, o contexto de produção, a intenção comunicativa percebidas nas palavras e nas informações paralinguísticas.

\section{REFERÊNCIAS}

RPI Revista de Pesquisa Interdisciplinar, Cajazeiras, v. 1, Ed. Especial, 381 - 389, set/dez. de 2016. 
ALMEIDA, I. D. [et. al.]. Tecnologias e educação: o uso do Youtube na sala de aula. In: Congresso Nacional de Educação, 2., 2016. Campina Grande. Anais. Disponível em: < http://www.editorarealize.com.br/revistas/conedu/anais.php>. Acesso em: 27 ago. 2016.

DELL'ISOLA, R. L. P. Retextualização de gêneros escritos. Rio de Janeiro: Lucerna, 2007.

KLEIMAN, A. B. Letramento e suas implicações para o ensino de língua materna. Signo, Santa Cruz do Sul, v. 32, n. 53, p. 1-25, dez. 2007.

KARNOPP. L. B; KLEIN, M.; LUNAZZI-LANZARIN, M. L. Produção, circulação e consumo da cultura surda brasileira. In: KARNOPP. L. B; KLEIN, M.; LUNAZZILANZARIN, M. L.(Orgs). Cultura surda na contemporaneidade: negociações, intercorrências e provocações. Canoas: Ed. ULBRA, 2011.

PINHEIRO, D. Produções surdas no YouTube: produzindo cultura. In: KARNOPP. L. B; KLEIN, M.; LUNAZZI-LANZARIN, M. L.(Orgs). Cultura surda na contemporaneidade: negociações, intercorrências e provocações. Canoas: Ed. ULBRA, 2011.

ROJO, R. H. R,. Protótipos didáticos para os multiletramentos. In: ROJO, R.H.R.;

MOURA, E. (Orgs). Multiletamentos na Escola.São Paulo: Parábola Editorial, 2012, p. 7-9.

SCHALLENBERGER, A. Comunidades surdas nas redes sociais: pela resistência e perpetuação da diferença através do humor. In: PERLIN, G.

SANTOS, R. C.; COSTA, G. B. Estrutura fonológica da Língua Brasileira de Sinais e da Língua Portuguesa: questões sobre a (in)dependência na estrutura linguística. Cadernos do CNLF, Vol. XVI, $\mathrm{N}^{\mathrm{O}} 04, \quad$ t. $2 . \quad$ Disponível em: < http://www.filologia.org.br/xvi_cnlf/tomo_2/169.pdf> Acesso em: 27 ago 2016.

SKLIAR, C. Perspectivas políticas e pedagógicas da educação bilíngue para surdos. In: SILVA, S.; VIZIN, M. (Orgs). Educação Especial, múltiplas leituras e diferentes significados. Campinas: Mercado das Letras, 2001.

YOUTUBE. A História do Youtube: The Youtube History - WebTV Joinville - HD - Parte 1.avi. Academia Joinvilense de Música. 2011. Disponível em:< https://www.youtube.com/watch?v=XZmlATVOIbg > . Acesso em: 02 de set de 2016.

RPI Revista de Pesquisa Interdisciplinar, Cajazeiras, v. 1, Ed. Especial, 381 - 389, set/dez. de 2016. 